\title{
PHYSICAL QUANTITIES AND UNITS
}

\author{
A. P. Sebekin and P. N. Selivanov \\ D. I. Mendeleyev Institute for Metrology(VNIIM), St. Petersburg, Russia
}

Keywords: physical quantity, units, dimensions, systems, equations, SI, natural systems.

\section{Contents}

1. Introduction

2. Dimension of Physical Quantity and its Value

3. Units of Physical Quantities: Systems of Quantities and Systems of Units

4. Equations Between Quantities

5. Dimensions of Physical Quantities

6. Le Système International d'Unités (SI)

6.1 General Characteristic of the SI

6.2 SI Base Units

6.3 SI Derived Units

6.4 Decimal Multiples and Submultiples of SI Units

7. Systems of Units of Physical Quantities

7.1 The Gaussian System

7.2 The CGS System

7.3 The MKS System

7.4 Systems of Units of Electromagnetic Quantities

7.4.1 The Electrostatic System of Units (CGSE)

7.4.2 The Electromagnetic System of Units (CGSM)

7.4.3 The Symmetric System of Units (CGS System)

7.4.4 The Practical System of Electric Units

7.5 The MKSA System

8. Natural Systems of Units

9. Conclusion

Glossary

Bibliography

Biographical Sketches

\section{Summary}

The paper presents a description of the importance of a system of physical quantities and units and the niche that it occupies in everyday life. Up-to-date terminology, definitions, and symbols are here given. Particular attention has been given to the concepts "physical quantity," “dimension and value of physical quantity," and "unit of physical quantity." The currently available systems of physical quantities and units with their symbols have been described. Physical quantities and their units that are regulated by the Système International d’Unités (SI) have been carefully considered.

In order to provide for the unification of units of physical quantities throughout the world, SI units are recommended for use in science, commerce, and technology. They 
are agreed internationally, accepted by the CGPM, and provide a reference in terms of which all other units are presently being defined. The SI base units and SI derived units, including those with special names, have an important advantage because they form a coherent system, that is, a system of units related by the rules of multiplication and division with a numerical factor equal to 1 . The paper also suggests the rules of forming coherent derived SI units.

The Système International d’Unités is a direct descendant of the decimal metric system, because it was based on a decimal number system and retained the principle of forming decimal multiples and submultiples of units from the SI units.

Besides, the first six prefixes of the Metric System entered into the SI prefixes. This paper gives the existing SI prefixes used to form multiples and submultiples of units based on the SI units. The multiples and submultiples of the SI units are also recommended for use in practice; they also relate to SI units.

Some non-SI units are still widely used in scientific, technical, and commercial literature and some will probably continue to be used for years. Other non-SI units, such as units of time, are so widely used in everyday life and so deeply rooted in the history and culture of mankind that they will continue to be used in the immediate future.

The paper presents the following categorization of the non-SI units: units accepted for use with the SI and those used temporarily with the SI.

In addition to the non-SI units, relative and logarithmic quantities, which are identical within all systems of units, are of considerable current use.

Unification of physical quantities used in different fields of physics follows the methods given in International Standard ISO 31. The systems of physical quantities presented in this document are also considered in the given paper. For each physical quantity, we indicate its name, symbol, definition, and an SI unit (with its name, symbol, and definition).

\section{Introduction}

Cognition of the surrounding world rests on information about physical quantities. Whatever area of activities we engage in, (science, industry, agriculture, and so on) we constantly face the necessity of measuring such physical quantities as length, mass, time, temperature, electrical and magnetic quantities, quality parameters of products, and so on. Measurements give a profound quantitative characteristic of different physical phenomena and allow us to apply them in everyday life.

Scientific investigations search for new laws of nature, from investigations of microand macrocosms, studies of the ocean and outer space, to investigations of substances and materials research in medicine and biology. All these are unimaginable without accurate measurements with which quantitative relationships between the phenomena studied are determined. 
In addition, no technological process can do without measurements; for example, serial production, a heart of today's industry, is based on the use of interchangeable parts whose dimensions are measured with a high degree of accuracy.

Measurements of different physical quantities are carried out in the course of a variety of tests and verifications, in certification of products, in environmental protection, in ensuring security of labor, and so on.

The concept "physical quantity" was first used in physics. Investigations of physical phenomena and the links between them, governed by laws, have necessitated the introduction into science of the concept of a considerable number of different quantities characterizing either special properties of matter or some peculiarities of the phenomena as such. These quantities have been named physical quantities.

Physical quantities are widely used in science because they allow us to represent the phenomena being studied in terms of mathematical relations and to obtain objective information that characterizes them both quantitatively and qualitatively.

\section{Dimension of Physical Quantity and its Value}

Presently, a physical quantity is defined as an attribute of a physical body (system, phenomenon, or process) that may be common for many physical bodies in a qualitative sense, while, in a quantitative sense, it is unique for each of them. In a qualitative sense, a physical quantity may be inherent in different material bodies, processes, or phenomena.

A physical quantity determined quantitatively is an individual property of a concrete object. For example, one may distinguish length, width, and height of an object. With knowledge of length, width, and height one can form an opinion concerning the distinctions between objects.

The concept "physical quantity" is used not only in physics but also in chemistry and in other sciences when, in order to evaluate an attribute of an object quantitatively, application of physical (experimental) methods becomes necessary.

It is obvious that any object has its own volume, mass, and so on. To put it otherwise, every object has its own quantitative definition; that is why one can distinguish objects by a dimension of one or other physical quantity. The concept "dimension" of a physical quantity is taken to mean quantitative definiteness of the quantity, being peculiar to any concrete material object, system, phenomenon, or process. The value of a physical quantity is an expression of the dimension of the physical quantity in terms of a certain number of units accepted for it. A numerical value of a physical quantity should not be confused with its dimension. A dimension of a physical quantity of a given object exists in reality irrespective of whether we know it or not. However, a numerical value of a physical quantity appears only once a dimension of a quantity of a certain object has been expressed with the help of a unit. 
A numerical value of a physical quantity is obtained from its measurement or calculation.

\section{Units of Physical Quantities: Systems of Quantities and Systems of Units}

For each physical quantity, one chooses a unit that does not differ from a corresponding quantity physically but has a quite definite dimension. Every concrete quantity can be defined as the product of a unit multiplied by an abstract number:

$X=\{X\}[X]$

where:

- $X$ is the value of the physical quantity;

- $\{X\}$ is the numerical value (abstract number); and

- $[X]$ is the unit of the physical quantity $X$.

This notation has been recommended by International Standard ISO 31/0.

Objective quantitative information about physical quantities can be obtained if the quantities are expressed through the units universally accepted for use.

Units of the Système International d'Unités (SI) adopted at the eleventh Conférence Générale des Poids et Mesures (CGPM) in 1960, and modified at subsequent meetings of the CGPM, have been used throughout the world in accordance with International Standard ISO 1000-(1992).

Before proceeding to a presentation of the structure and content of the SI, consider the system of physical quantities that is appropriate to the SI.

By a system of physical quantities we mean a total combination of physical quantities formed in keeping with widely accepted principles; some quantities are taken as independent (base quantities) and the others are defined as functions of the independent quantities and are called "derived" quantities.

The system of quantities upon which the international system of units has been based has seven base quantities. It is called the "LMTIQNJ" system of quantities, that is, length $\mathrm{L}$, mass $\mathrm{M}$, time $\mathrm{T}$, electric current $\mathrm{I}$, thermodynamic temperature $\mathrm{Q}$, amount of substance $\mathrm{N}$ and luminous intensity $\mathrm{J}$. In addition to the base quantities, the system also includes derived physical quantities that are related to the base quantities by defining equations

With advances in physics and metrology, the system of physical quantities forming the basis of the Système International d'Unités has been gradually developed. It is subdivided into the following quantity systems:

- mechanics (LMT), in the defining equations of which the usual three base quantities (length, mass, and time) are used;

- electricity and magnetism (LMTI) with four base quantities (length, mass, time, and electric current); 
- thermodynamics (LMTQ);

- light and related electromagnetic radiations (LMTJ);

- physical chemistry and molecular physics (LMTQN); and

- ionizing radiations (LMTIN).

Symbols assigned to the base quantities are used to denote quantity systems; for example, the quantity system for mechanics in which length $\mathrm{L}$, mass $\mathrm{M}$, and time $\mathrm{T}$ are accepted for use as the base quantities is called the LMT system.

\section{Equations Between Quantities}

Coupling equations relating the quantities concerned can reflect the laws of nature and (or) be the defining equations for derived quantities. In these equations, letter symbols represent physical quantities. For example, consider the equation:

$V=l / t$

where:

- $\quad v$ is the velocity of a point moving linearly and uniformly;

- $l$ is the length or distance moved; and

- $t$ is the time taken to move that distance.

This equation reflects the existing dependence of the velocity on the distance and time and is a defining equation of the physical quantity (velocity) in the LMT system of quantities.

Eq. (2) is a defining equation presented in the simplest algebraic form. With the proviso that the algebraic form is kept, the equation defining a certain derived quantity $X$, can be written in a general form:

$$
X=Z X_{1}^{\alpha 1} X_{2}^{\alpha 2} \ldots X_{n}^{\alpha n}
$$

where:

- $X_{1}, \ldots, X_{n}$ are the base quantities or some other already known derived quantities through which the quantity $X$ is determined;

- $\alpha 1, \ldots \ldots, \alpha n$ are abstract numbers; and

- $Z$ is a coefficient.

There are also some other, more complicated defining equations that can take differential, integral, exponential, and other forms.

\section{Dimensions of Physical Quantities}

Derived physical quantities can be both dimensional and dimensionless. A dimension of a physical quantity is an expression in the form of a power monomial that is composed of the products of symbols for base physical quantities raised to different powers. The expression reflects the relationship between a given physical quantity and physical 
quantities taken for the base ones in the given system of quantities, which has a proportionality coefficient equal to 1 .

The concept "dimension" also spreads to the base quantities. The dimension of a base quantity in respect to itself is equal to 1 and does not depend on other quantities; that is, the formula for the dimension of the base quantity coincides with its symbol. For example, a dimension of length is $\mathrm{L}$, that of mass is $\mathrm{M}$, and that of time is $\mathrm{T}$, and so on.

In accordance with International Standard ISO 31/0, the dimension of quantities is designated as “dim” (abbreviation of “dimension”).

To find a dimension of a derived physical quantity in a certain quantity system, it is necessary that the dimensions of quantities should be substituted for their symbols to the right side of the defining equation of this quantity. For example, in the LMT system for Eq. (2) we obtain the following:

$$
\operatorname{dim} v=\mathbf{L M}^{0} \mathbf{T}^{-1}=\mathbf{L} \mathbf{T}^{-1}
$$

The exponents to which the dimensions of the base physical quantities were raised in Eq. (4) - $(+1)$ for L, 0 for M, $(-1)$ for T-are called the exponents of the dimension of the physical quantity. They may take different values, that is, they may be integer or fractional, positive or negative, and may be equal to 0 .

A dimension of any derived physical quantity in the LMT system of quantities may be expressed by an exponential monomial:

$\operatorname{dim} X=\mathrm{L}^{\alpha} \mathrm{M}^{\beta} \mathrm{T}^{\gamma}$

A dimension of a derived physical quantity in a system of quantities corresponding to the Système International d'Unités (SI) that is based on quantities such as length, mass, time, electric current, thermodynamic temperature, luminous intensity, and amount of substance can be expressed, in a general form, as:

$\operatorname{dim} X=\mathrm{L}^{\alpha} \mathrm{M}^{\beta} \mathrm{T}^{\gamma} \mathrm{I}^{\delta} \mathrm{Q}^{\varepsilon} \mathrm{J}^{\rho} \mathrm{N}^{q}$

In Eqs. (5) and (6), $\alpha, \beta, \gamma, \ldots \rho, q$ are the exponents of a dimension of a derived physical quantity in the LMTIQJN system of quantities.

Thus, physical quantities in the dimensions of which any one of the base physical quantities is raised to a power not equal to zero refer to derived physical quantities.

Dimensionless physical quantities are those in the dimensions of which base physical quantities enter with exponents equal to zero.

Dimensionless quantities refer to ratios of two quantities of the same kind, that is, relative quantities or their functions (exponents, products of exponents, logarithms, trigonometric functions, and so on). Examples of relative quantities are relative 
lengthening, efficiency, magnetic permeability, and so on. The dimension of a derived physical quantity is also the dimension of its unit. Mention may be also made of a number of practical applications of the concept "dimension of physical quantity."

Firstly, it is possible, by reference to the dimension of a quantity, to determine by how much the dimension of a unit of a given derived physical quantity would vary under a change of the dimensions of the units of the quantities taken for the base ones.

Secondly, by means of the dimensions of physical quantities, one can check the correctness of equations resulting from theoretical conclusions, by using the principle of dimensional similarity of the terms of physical equations. Thus, the dimensions on the right and left hand sides of the equation linking different physical quantities should be identical.

Analysis of dimensions and the theory of physical similarity, which allow us to study complicated physical phenomena using reduced models, are also based on the principle of dimensional similarity of the terms of physical equations.

\section{Le Système International d'Unités (SI)}

\subsection{General Characteristic of the SI}

By the turn of the eighteenth century, the growth in the world's industry and commerce generated the need to establish a unified system of measures that could serve "à tous les temps à tous les peuples.”

French scientists created the decimal metric system, based on a single unit, a unit of length, the meter, which was originally intended to be one forty millionth part of Earth's meridian. The units of area and volume were defined as the square meter and the cubic meter, respectively, and the unit of mass, the kilogram, as the mass equal to the mass of a cubic decimeter of pure water at $4{ }^{\circ} \mathrm{C}$.

The decimal metric system was used to create multiples and submultiples of all derived units and of the base unit, the meter, as well; factors equal to $10^{n}$ were used, where $n$ is a positive or negative integer number.

A series of the following first six prefixes were introduced, namely, deca $\left(10^{1}\right)$, hecto $\left(10^{2}\right)$, kilo $\left(10^{3}\right)$, deci $\left(10^{-1}\right)$, centi $\left(10^{-2}\right)$ and milli $\left(10^{-3}\right)$.

The decimal metric system adopted in France at the end of the eighteenth century gained recognition in the second part of the nineteenth century. In this connection, a demand arose for a standing international organization, whose principal task was to use the metric system and to contribute to its further improvement and propagation.

In Paris; at the international diplomatic conference held in May 20, 1875, representatives of seventeen nations signed the Convention du Mètre, in accordance with which the Bureau International des Poids et Mésures (BIPM) was established for the following purposes: 
- To maintain the international prototypes of the meter and the kilogram.

- To carry out (at regular intervals) comparisons of national prototypes of the meter and kilogram against their international prototypes.

- To modify the metric system.

The Convention du Mètre was revised according to the international agreement signed in Sèvres, at the BIPM, in October 6, 1921, with the aim to extend the scope and responsibilities of the convention to the field of electric measurements and determination of fundamental physical constants.

Further investigations at the BIPM were aimed at improvement and modification of the metric system in order to establish a practical international system of units, which would involve all kinds of measurements. It is worth noting that, in accordance with the Convention du Mètre, the Conférence Générale des Poids et Mésures (CGPM) has become the world's highest authority on units of physical quantities, on their definitions as well as on the methods for reproduction of units by means of measurement standards.

Some of the questions considered are being settled by the Comite International des Poids et Mésures (CIPM) on behalf of the Conférence Générale des Poids et Mésures (CGPM). The proceedings of the CIPM are recorded in protocols of its meetings, that is, in the Procès-Verbaux des Séances du CIPM (PV), published by the BIPM, and the official reports of the CGPM are annually published by the BIPM in the Comptes Rendus des Séances de la Conférence Générale des Poids et Mésures (CR).

The name "Système International d'Unités (SI) was given to this new practical system by the eleventh CGPM (Resolution 12), in 1960.

The resolutions of the CGPM cited in this text are presented in the brochure of the BIPM entitled Le Système International d'Unités, Appendix 1.

The International System of units meets the following requirements, which have been used as the basis for its establishment:

- Possibility of assuring the unification of units of physical quantities in all countries throughout world.

- Versatility, which means that the system is useful for all fields of human activity.

- Uniqueness of a unit for each physical quantity.

- Consistency (coherence), which is achieved by a choice of a small number of base units while derived units are formed with the use of defining equations with coefficients equal to 1 .

- Possibility of assuring high accuracy in realization of units experimentally (base units first).

Units of the Système International d'Unités (SI) can be divided into two classes: SI base units and SI derived units. 


\section{Bibliography}

Bureau International des Poids et Mésures (BIPM) (1987). Le BIPM et la Convention du Mètre, 45 pp. Sèvres, Paris: BIPM. [This describes the BIPM experimental basis used for carrying out investigations on further improvement of the Système International d'Unités.]

Bureau International des Poids et Mésures (BIPM) (1998). Le Système International d'Unités (SI), 152 pp. Sèvres, Paris: BIPM. [This publication is a guide to the International System of Units (SI) used throughout the world.]

Convention du Mètre (signed 20 May 1875). Paris: Gautier-Villars, imprimeur-librair (1905). 17 pp. [In accordance with this document, the decimal metric system adopted in 1875, and the Système International d'Unités approved later, in 1960, were recommended for use throughout the world.]

International Council for Science (CODATA) (1986). The 1986 Adjustment of the Fundamental Physical Constants, 36 pp. A Report of the CODATA Task Group on Fundamental Constants, CODATA Bulletin No. 63, November 1986. [This contains the agreed values of the fundamental physical constants recommended for use in different fields of science, technology, and education throughout the world.]

International Organization of Legal Metrology (OIML) (1978). Vocabulaire de Mètrologie Legale, 184 pp. OIML. [This presents terms and definitions of concepts used in the field of legal metrology.]

International Standards Organization (1992a). International Standard IEC 27-1. Letter symbols to be used in electrical engineering. Part 1, $111 \mathrm{pp.} \mathrm{General,} \mathrm{IEC.} \mathrm{[This} \mathrm{IEC} \mathrm{document} \mathrm{is} \mathrm{aimed} \mathrm{at} \mathrm{unification} \mathrm{of}$ physical quantities and units used in electrical and electronic engineering.]

International Standards Organization (1992b).International Standard ISO 31-0. Quantities and Units, Part 0: General Principles, 37 pp. ISO. [The standard contains data on general rules and regulations concerning physical quantities, equations, and units with their symbols as well as rules of writing symbols and numbers.]

International Standards Organization (1992c). International Standard ISO 31 (Parts 0-13). Quantities and Units, 266 pp. ISO. [This is a fundamental international documentary standard on problems of unification of physical quantities and units used in different fields of physics, and on problems of unification of mathematical signs and symbols used in different areas of science and technology.]

International Standards Organization (1992d). International Standard ISO 1000. SI Units and recommendations for the use of their multiples and certain other units, $22 \mathrm{pp}$. ISO. [This document is a fundamental international documentary standard according to which the Système International d'Unités is being introduced throughout the world. This also contains the recommended multiples and submultiples of the SI units.]

International Standards Organization (1993). Congress International Vocabulary of Basic and General Terms in Metrology (VIM), $59 \mathrm{pp}$. [The vocabulary is a fundamental international documentary standard on terminology in the field of metrology.]

\section{Biographical Sketches}

Alexei P. Sebekin

Date of birth: May 27, 1945. Education: In 1968, A. Sebekin graduated from the Leningrad Electrotechnical Institute named after V. I. Ul'yanov (Lenin), the Faculty of Electronic Engineering. In 
1981, he got the academic degree of Candidate and in 1995, he was elected corresponding member of the Metrological Academy of the Russian Federation. Now he is academic secretary of the Coordination Academic Council of the Metrological Academy on fundamental problems of metrology, and academic secretary of the Section "Problems of Metrology" of the Academic and Technical Council of the GOSSTANDART of Russia. Since 1968, he has been with the D. I. Mendeleyev Institute for Metrology, and now is head of the Research Department of General Metrology. A. Sebekin is member of TC 25/WG of the MEC: "Quantities, Units, and their Letter Symbols”

Academic rank: Senior Scientist.

Sphere of research interests: Theoretical and legal metrology.

Publications: 50 .

Petr N. Selivanov

Date of birth: 1929. Education: In 1957, P. Selivanov graduated from the Leningrad Higher Marine Engineering School named after Stepan O. Makarov. Since 1969, he has been with the D. I. Mendeleyev Institute for Metrology and, in 1967, got the academic degree of Candidate. In 1968, he was conferred the rank of Senior Scientist.

Sphere of research interests: Theoretical and methodical problems of unification of physical quantities and units, and the problems of international standardization.

Publications: 47. 